\title{
Ranking Fuzzy Numbers with a Distance Method using Circumcenter of Centroids and an Index of Modality
}

\author{
P. Phani Bushan Rao ${ }^{1}$ and N. Ravi Shankar ${ }^{2}$ \\ ${ }^{1}$ Department of Mathematics, GITAM Institute of Technology, GITAM University, Visakhapatnam 530045, India \\ ${ }^{2}$ Department of Applied Mathematics, GITAM Institute of Science, GITAM University, Visakhapatnam 530045, India
}

Correspondence should be addressed to N. Ravi Shankar, drravi68@gmail.com

Received 14 December 2010; Revised 18 April 2011; Accepted 26 April 2011

Academic Editor: José Luis Verdegay

Copyright (C) 2011 P. P. B. Rao and N. R. Shankar. This is an open access article distributed under the Creative Commons Attribution License, which permits unrestricted use, distribution, and reproduction in any medium, provided the original work is properly cited.

\begin{abstract}
Ranking fuzzy numbers are an important aspect of decision making in a fuzzy environment. Since their inception in 1965, many authors have proposed different methods for ranking fuzzy numbers. However, there is no method which gives a satisfactory result to all situations. Most of the methods proposed so far are nondiscriminating and counterintuitive. This paper proposes a new method for ranking fuzzy numbers based on the Circumcenter of Centroids and uses an index of optimism to reflect the decision maker's optimistic attitude and also an index of modality that represents the neutrality of the decision maker. This method ranks various types of fuzzy numbers which include normal, generalized trapezoidal, and triangular fuzzy numbers along with crisp numbers with the particularity that crisp numbers are to be considered particular cases of fuzzy numbers.
\end{abstract}

\section{Introduction}

Ranking fuzzy numbers are an important tool in decision making. In fuzzy decision analysis, fuzzy quantities are used to describe the performance of alternatives in modeling a real-world problem. Most of the ranking procedures proposed so far in the literature cannot discriminate fuzzy quantities and some are counterintuitive. As fuzzy numbers are represented by possibility distributions, they may overlap with each other, and hence it is not possible to order them. It is true that fuzzy numbers are frequently partial order and cannot be compared like real numbers which can be linearly ordered. In order to rank fuzzy quantities, each fuzzy quantity is converted into a real number and compared by defining a ranking function from the set of fuzzy numbers to a set of real numbers which assign a real number to each fuzzy number where a natural order exists. Usually by reducing the whole of any analysis to a single number, much of the information is lost and hence an attempt is to be made to minimize this loss. Various ranking procedures have been developed since 1976 when the theory of fuzzy sets were first introduced by Zadeh [1]. Ranking fuzzy numbers were first proposed by Jain [2] for decision making in fuzzy situations by representing the ill-defined quantity as a fuzzy set. Since then, various procedures to rank fuzzy quantities are proposed by various researchers. Bortolan and Degani [3] reviewed some of these ranking methods [2, 4-14] for ranking fuzzy subsets. Chen [15] presented ranking fuzzy numbers with maximizing set and minimizing set. Dubois and Prade [16] presented the mean value of a fuzzy number. Lee and Li [17] presented a comparison of fuzzy numbers based on the probability measure of fuzzy events. Delgado et al. [18] presented a procedure for ranking fuzzy numbers. Campos and Muñoz [19] presented a subjective approach for ranking fuzzy numbers. Kim and Park [20] presented a method of ranking fuzzy numbers with index of optimism. Yuan [21] presented a criterion for evaluating fuzzy ranking methods. Heilpern [22] presented the expected value of a fuzzy number. Saade and Schwarzlander [23] presented ordering fuzzy sets over the real line. Liou and Wang [24] presented ranking fuzzy numbers with integral value. Choobineh and Li [25] presented an index for ordering fuzzy numbers. Chang and Lee [26] presented ranking of fuzzy sets based on the concept of existence. Since then several methods 
have been proposed by various researchers which includes ranking fuzzy numbers using area compensation, distance method, maximizing and minimizing set, decomposition principle, and signed distance [27-30]. Wang and Kerre $[31,32]$ classified all the above ranking procedures into three classes. The first class consists of ranking procedures based on fuzzy mean and spread $[6,8-11,19,24,25,27]$, and second class consists ranking procedures based on fuzzy scoring $[2,5,12,15,20,33]$, whereas the third class consists of methods based on preference relations $[4,7,13,18,21$, $23,34,35]$ and concluded that the ordering procedures associated with first class are relatively reasonable for the ordering of fuzzy numbers specially the ranking procedure presented by Adamo [9] which satisfies all the reasonable properties for the ordering of fuzzy quantities. The methods presented in the second class are not doing well and the methods [21, 23, 34, 35] which belong to class three are reasonable. Later on, ranking fuzzy numbers by preference ratio [36], left and right dominance [37], fuzzy distance measure [38], area between the centroid point and original point [39], preference weighting function expectations [40], sign distance [41], fuzzy simulation analysis method [42], an area method using radius of gyration [43], distance minimization [44], and fuzzy risk analysis based on the ranking of generalized trapezoidal fuzzy numbers [45]. Garcia and Lamata [46] modified the index of Liou and Wang [24] for ranking fuzzy numbers. The development in ranking fuzzy numbers can also be found in [47-58]. Most of the methods presented above cannot discriminate fuzzy numbers, and some methods do not agree with human intuition, whereas some methods cannot rank crisp numbers which are a special case of fuzzy numbers.

In this paper, a new method is proposed which is based on Circumcenterr to rank fuzzy quantities. In a trapezoidal fuzzy number, first the trapezoid is split into three parts where the first, second, and third parts are a triangle, a rectangle, and a triangle respectively. Then the Centroids of these three parts are calculated followed by the calculation of the Circumcenterr of these Centroids. Finally, a ranking function is defined which is the Euclidean distance between the Circumcenterr point and the original point to rank fuzzy numbers. Most of the ranking procedures proposed in the literature use Centroid of trapezoid as reference point, as the Centroid is a balancing point of the trapezoid. But the Circumcenterr of Centroids can be considered a much more balancing point as this point is equidistant from all the vertices which are Centroids. Further, this method uses an index of optimism to reflect the decision maker's optimistic attitude and also uses an index of modality that represent the neutrality of the decision maker.

The work is organized as follows. Section 2 briefly introduces the basic concepts and definitions of fuzzy numbers. Section 3 presents the proposed new method. In Section 4, the proposed method has been explained with examples which describe the advantages and the efficiency of the method which ranks generalized fuzzy numbers, images of fuzzy numbers, and even crisp numbers. In Section 5, the method demonstrates its robustness by comparing with other methods like Liou and Wang, Yager, and others where the methods cannot discriminate fuzzy quantities and do not agree with human intuition. Finally, the conclusions of the work are presented in Section 6.

\section{Preliminaries}

Definition 1 (Fuzzy Numbers and Membership Functions). A fuzzy number $\tilde{A}$ is a fuzzy subset in support $\mathbb{R}$ (real number) which is both "normal" and "convex" with membership function

$$
f_{\widetilde{A}}(x)= \begin{cases}f_{\widetilde{A}}^{L}(x), & a \leq x \leq b, \\ w, & b \leq x \leq c, \\ f_{\widetilde{A}}^{R}(x), & c \leq x \leq d, \\ 0, & \text { otherwise }\end{cases}
$$

where $0<w \leq 1$ is a constant, $a, b, c, d$ are real numbers, and $f_{\widetilde{A}}^{L}:[a, b] \rightarrow[0, w], f_{\widetilde{A}}^{R}:[c, d] \rightarrow[0, w]$ are two strictly monotonic and continuous functions from $\mathbb{R}$ to the closed interval $[0, w]$. It is customary to write a fuzzy number as $\widetilde{A}=$ $(a, b, c, d ; w)$. If $w=1$, then $\widetilde{A}=(a, b, c, d ; 1)$ is a normalized fuzzy number, otherwise $\tilde{A}$ is said to be a generalized or nonnormal fuzzy number.

If the membership function $f_{\widetilde{A}}(x)$ is piecewise linear, then $\tilde{A}$ is said to be a trapezoidal fuzzy number. The membership function of a trapezoidal fuzzy number is given by

$$
f_{\widetilde{A}}(x)= \begin{cases}\frac{w(x-a)}{b-a}, & a \leq x \leq b, \\ w, & b \leq x \leq c, \\ \frac{w(x-d)}{c-d}, & c \leq x \leq d, \\ 0, & \text { otherwise. }\end{cases}
$$

If $w=1$, then $\tilde{A}=(a, b, c, d ; 1)$ is a normalized trapezoidal fuzzy number and $\tilde{A}$ is a generalized or nonnormal trapezoidal fuzzy number if $0<w<1$. The image of $\widetilde{A}=(a, b, c, d ; w)$ is given by $-\widetilde{A}=(-d,-c,-b,-a ; w)$.

As a particular case if $b=c$, the trapezoidal fuzzy number reduces to a triangular fuzzy number given by $\tilde{A}=$ $(a, b, d ; w)$. The value of " $b$ " corresponds with the mode or core and $[a, d]$ with the support. If $w=1$, then $\tilde{A}=(a, b, d)$ is a normalized triangular fuzzy number $\tilde{A}$ is a generalized or nonnormal triangular fuzzy number if $0<w<1$.

As $f_{\widetilde{A}}^{L}(x):[a, b] \rightarrow[0, w]$ and $f_{\widetilde{A}}^{R}(x):[c, d] \rightarrow[0, w]$ are strictly monotonic and continuous functions, their inverse functions $g_{\widetilde{A}}^{L}(y):[0, w] \rightarrow[a, b]$ and $g_{\widetilde{A}}^{R}(y):[0, w] \rightarrow[c, d]$ are also continuous and strictly monotonic. Hence $g_{\widetilde{A}}^{L}(y)$ and $g_{\widetilde{A}}^{R}(y)$ are integrable on $[0, w]$.

Definition 2 (Liou and Wang's Ranking Method). Liou and Wang [24] ranked fuzzy numbers with total integral value. For a fuzzy number defined by Definition 1, the total integral value is defined as $I_{T}^{\alpha}(\tilde{A})=\alpha I_{R}(\tilde{A})+(1-\alpha) I_{L}(\tilde{A})$ where 


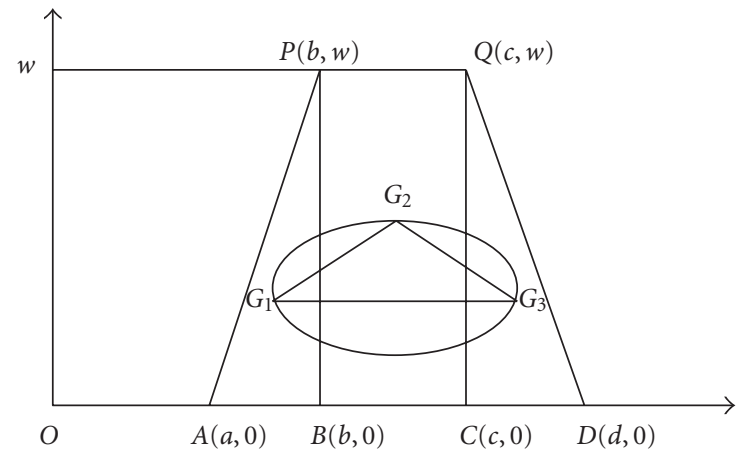

FIgURE 1: Circumcenterr of Centroids.

$I_{R}(\widetilde{A})=\int_{0}^{1} g_{\widetilde{A}}^{R}(y) d y$ and $I_{L}(\widetilde{A})=\int_{0}^{1} g_{\tilde{A}}^{L}(y) d y$ are the right and left integral values of $\widetilde{A}$, respectively, and $\alpha \in[0,1]$ is the index of optimism which represents the degree of optimism of a decision maker. If $\alpha=0$, the total integral value represents a pessimistic decision maker's view point which is equal to left integral value. If $\alpha=1$, the total integral value represents an optimistic decision maker's view point and is equal to the right integral value and when $\alpha=0.5$, the total integral value represents an moderate decision maker's view point and is equal to the mean of right and left integral values. For a decision maker, the larger the value of $\alpha$ is, the higher is the degree of optimism.

Definition 3 (Garcia and Lamata's Ranking Method). Garcia and Lamata [46] modified the index of Liou and Wang [24] for ranking fuzzy numbers. This method use an index of optimism to reflect the decision maker's optimistic attitude, which is not enough to discriminate fuzzy numbers, but rather it also uses an index of modality that represents the neutrality of the decision maker. For a fuzzy number defined by Definition 1, Garcia and Lamata [46] proposed an index associated with the ranking as the convex combination $I_{\beta, \alpha}(\widetilde{A})=\beta S_{M}(\widetilde{A})+(1-\beta) I_{T}^{\alpha}(\widetilde{A})$, where $S_{M}(\widetilde{A})$ is the area of the core of the fuzzy number which is equal to " $b$ " for a triangular fuzzy number defined by $\tilde{A}=(a, b, d ; w)$ and the average value of the plateau in case of a trapezoidal fuzzy number given by $\widetilde{A}=(a, b, c, d ; w), \beta \in[0,1]$ is the index of modality that represents the importance of central value against the extreme values, $\alpha \in[0,1]$ is the degree of optimism of the decision maker, and $I_{T}^{\alpha}(\widetilde{A})$ has its own meaning as defined in Definition 2.

\section{Proposed Method}

Definition 4. The Centroid of a trapezoid is considered as the balancing point of the trapezoid (Figure 1). Divide the trapezoid into three plane figures. These three plane figures are a triangle $(A P B)$, a rectangle $(B P Q C)$, and again a triangle $(C Q D)$, respectively. The Circumcenterr of the Centroids of these three plane figures is taken as the point of reference to define the ranking of generalized fuzzy numbers. The reason for selecting this point as a point of reference is that each Centroid point ( $G_{1}$ of triangle $A P B, G_{2}$ of rectangle $B P Q C$, and $G_{3}$ of triangle $C Q D$ ) are balancing points of each individual plane figure, and the Circumcenterr of these Centroid points is equidistant from each vertex (which are Centroids). Therefore, this point would be a better reference point than the Centroid point of the trapezoid.

Consider a generalized trapezoidal fuzzy number $\tilde{A}=$ $(a, b, c, d ; w)$ (Figure 1).

The Centroids of the three plane figures are $G_{1}=((a+$ $2 b) / 3, w / 3), G_{2}=((b+c) / 2, w / 2)$, and $G_{3}=((2 c+d) / 3, w / 3)$, respectively. Equation of the line $\overleftrightarrow{G_{1} G_{3}}$ is $y=w / 3$ and $G_{2}$ does not lie on the line $\overleftrightarrow{G_{1} G_{3}}$. Therefore, $G_{1}, G_{2}$ and $G_{3}$ are non-collinear and they form a triangle.

We define the Circumcenterr $S_{\widetilde{A}}\left(\overline{x_{0}}, \overline{y_{0}}\right)$ of the triangle with vertices $G_{1}, G_{2}$ and $G_{3}$ of the generalized trapezoidal fuzzy number $\tilde{A}=(a, b, c, d ; w)$ as

$$
\begin{aligned}
& S_{\widetilde{A}}\left(\overline{x_{0}}, \overline{y_{0}}\right) \\
& \quad=\left(\frac{a+2 b+2 c+d}{6}, \frac{(2 a+b-3 c)(2 d+c-3 b)+5 w^{2}}{12 w}\right) .
\end{aligned}
$$

As a special case, for triangular fuzzy number $\tilde{A}=(a, b, d ; w)$, that is, $c=b$ the Circumcenterr of Centroids is given by

$$
S_{\widetilde{A}}\left(\overline{x_{0}}, \overline{y_{0}}\right)=\left(\frac{a+4 b+d}{6}, \frac{4(a-b)(d-b)+5 w^{2}}{12 w}\right) .
$$

Definition 5. For a generalized trapezoidal fuzzy number $\tilde{A}=(a, b, c, d ; w)$, with Circumcenterr of Centroids $S_{\widetilde{A}}\left(\overline{x_{0}}, \overline{y_{0}}\right)$ defined by $(3)$, we define the index associated with the ranking as $I_{\alpha}(\widetilde{A})=\alpha \overline{y_{0}}+(1-\alpha) \overline{x_{0}}$ where $\alpha \in[0,1]$ is the index of optimism which represents the degree of optimism of a decision maker. If $\alpha=0$, we have a pessimistic decision maker's view point which is equal to the distance of the Circumcenterr from $y$-axis. If $\alpha=1$, we have an optimistic decision maker's view point and is equal to the distance of the Circumcenterr from $x$-axis, and when $\alpha=0.5$, we have the moderate decision maker's view point and is equal to the mean of the distances of Circumcenterr from $y$ and $x$ axes. The larger the value of $\alpha$ is, the higher the degree of optimism of the decision maker. The index of optimism is not alone sufficient to discriminate fuzzy numbers as this uses only the extreme values of the Circumcenterr of Centroids. Hence, we upgrade this by using an index of modality which represents the importance of central value along with index of optimism.

Definition 6. For a generalized trapezoidal fuzzy number $\tilde{A}=(a, b, c, d ; w)$, with Circumcenterr of Centroids $S_{\widetilde{A}}\left(\overline{x_{0}}, \overline{y_{0}}\right)$ defined by (3), we define the index associated with the ranking as $I_{\alpha, \beta}(\tilde{A})=\beta\left(\left(\overline{x_{0}}+\overline{y_{0}}\right) / 2\right)+(1-\beta) I_{\alpha}(\tilde{A})$ where $\beta \in[0,1]$ is the index of modality which represents the importance of central value against the extreme values $\overline{x_{0}}$ and $\overline{y_{0}}$ and $I_{\alpha}(\tilde{A})$ is the one which is defined in Definition 5. Here, $\beta$ represents the weight of the central value and $(1-\beta)$ is the weight associated with the extreme values $\overline{x_{0}}$ and $\overline{y_{0}}$. 
Definition 7. For any decision maker whether pessimistic ( $\alpha=0)$, optimistic $(\alpha=1)$, or neutral $(\alpha=0.5)$, the ranking function of the trapezoidal fuzzy number $\tilde{A}=(a, b, c, d ; w)$ which maps the set of all fuzzy numbers to a set of real numbers is defined as $R(\widetilde{A})=\sqrt{{\overline{x_{0}}}^{2}+{\overline{y_{0}}}^{2}}$ which is the Euclidean distance from the Circumcenterr of the Centroids as defined in Definition 4 and the original point.

Using the above definitions we define ranking between fuzzy numbers as follows

Let $\widetilde{A}$ and $\widetilde{A}_{j}$ two fuzzy numbers, then

(i) if $R\left(\widetilde{A}_{i}\right)>R\left(\widetilde{A}_{j}\right)$, then $\widetilde{A}_{i}>\widetilde{A}_{j}$,

(ii) if $R\left(\widetilde{A}_{i}\right)<R\left(\widetilde{A}_{j}\right)$, then $\widetilde{A}_{i}<\widetilde{A}_{j}$,

(iii) if $R\left(\widetilde{A}_{i}\right)=R\left(\widetilde{A}_{j}\right)$ then in this case the discrimination of fuzzy numbers is not possible. In such cases we use Definition 6 to rank fuzzy numbers as Definition 5 alone is not sufficient to discriminate in all cases, that is, if $I_{\alpha, \beta}\left(\tilde{A}_{i}\right)>I_{\alpha, \beta}\left(\widetilde{A}_{j}\right)$, then $\widetilde{A}_{i}>\tilde{A}_{j}$, and if $I_{\alpha, \beta}\left(\widetilde{A}_{i}\right)<I_{\alpha, \beta}\left(\widetilde{A}_{j}\right)$ then $\widetilde{A}_{i}<\widetilde{A}_{j}$.

\section{Examples}

Example 8. Let $\widetilde{A}=(0.1,0.3,0.3,0.5 ; 1), \widetilde{B}=(0.2,0.3,0.3$, $0.4 ; 1)$, and $\widetilde{C}=(1,1,1,1 ; 1)$.

Then, $S_{\widetilde{A}}\left(\overline{x_{0}}, \overline{y_{0}}\right)=(0.3,0.4033), S_{\widetilde{B}}\left(\overline{x_{0}}, \overline{y_{0}}\right)=(0.3,0.4133)$, and $S_{\tilde{C}}\left(\overline{x_{0}}, \overline{y_{0}}\right)=(1,0.4166)$.

Therefore, $R(\widetilde{A})=0.5026, R(\widetilde{B})=0.5107$, and $R(\widetilde{C})=$ $1.0833 \Rightarrow \widetilde{A}<\widetilde{B}<\widetilde{C}$.

It is observed that the above ranking order is unaltered even by using the index of modality proposed in Definition 6 whoever might be the decision maker.

Example 9. Let $\tilde{A}=(0.1,0.3,0.3,0.5 ; 1), \widetilde{B}=(-0.5,-0.3$, $-0.3,-0.1 ; 1)$.

Then, $S_{\widetilde{A}}\left(\overline{x_{0}}, \overline{y_{0}}\right)=(0.3,0.4033)$ and $S_{\widetilde{B}}\left(\overline{x_{0}}, \overline{y_{0}}\right)=(-0.3$, $0.4033)$

Therefore, $R(\widetilde{A})=0.5026, R(\widetilde{B})=0.5026$, and discrimination of fuzzy numbers is not possible by using Definition 7.

Now, by using Definition 6, we have the following.

(i) For a pessimistic decision make,

$$
\begin{gathered}
I_{0, \beta}(\widetilde{A})=0.35165 \beta+(1-\beta)(0.3), \\
I_{0, \beta}(\widetilde{B})=0.05165 \beta+(1-\beta)(-0.3) .
\end{gathered}
$$

As $0.35165 \beta+(1-\beta)(0.3)>0.05165 \beta+(1-\beta)$ $(-0.3) \Rightarrow \widetilde{A}>\widetilde{B}$

(ii) For a optimistic decision maker,

$$
\begin{aligned}
& I_{1, \beta}(\widetilde{A})=0.35165 \beta+(1-\beta)(0.4033), \\
& I_{1, \beta}(\widetilde{B})=0.05165 \beta+(1-\beta)(0.4033) .
\end{aligned}
$$

As $0.35165 \beta+(1-\beta)(0.4033)>0.05165 \beta+(1-\beta)$ $(0.4033) \Rightarrow \widetilde{A}>\widetilde{B}$. (iii) For a neutral decision maker,

$$
\begin{aligned}
& \quad I_{0.5, \beta}(\tilde{A})=0.35165 \beta+(1-\beta)(0.35165), \\
& \qquad I_{0.5, \beta}(\widetilde{B})=0.05165 \beta+(1-\beta)(0.05165) . \\
& \text { As } 0.35165 \beta+(1-\beta)(0.35165)>0.05165 \beta+(1-\beta) \\
& (0.05165) \Rightarrow \widetilde{A}>\widetilde{B} .
\end{aligned}
$$

Thus, we see that the ranking order is same in all the three cases.

Example 10. Let $\widetilde{A}=(0.1,0.3,0.3,0.5 ; 1)$ and $\widetilde{B}=(-0.5,-0.3$, $-0.3,-0.1 ; 1)$.

Then, $-\tilde{A}=(-0.5,-0.3,-0.3,-0.1 ; 1)$ and $-\widetilde{A}=(0.1$, $0.3,0.3,0.5 ; 1), S_{-\widetilde{A}}\left(\overline{x_{0}}, \overline{y_{0}}\right)=(-0.3,0.4033)$ and $S_{-\widetilde{B}}\left(\overline{x_{0}}, \overline{y_{0}}\right)=$ $(0.3,0.4033)$.

Therefore $R(-\widetilde{A})=0.5026, R(-\widetilde{B})=0.5026$, and discrimination of fuzzy numbers is not possible by using Definition 7.

Now, by using Definition 6 we have the following.

(i) For an pessimistic decision maker,

$$
\begin{gathered}
I_{0, \beta}(-\widetilde{A})=0.05165 \beta+(1-\beta)(-0.3), \\
I_{0, \beta}(-\widetilde{B})=0.35165 \beta+(1-\beta)(0.3) .
\end{gathered}
$$

As $0.35165 \beta+(1-\beta)(0.3)>0.05165 \beta+(1-\beta)$ $(-0.3) \Rightarrow-\tilde{A}<-\widetilde{B}$

(ii) For an optimistic decision maker,

$$
\begin{aligned}
& I_{1, \beta}(-\widetilde{A})=0.05165 \beta+(1-\beta)(0.4033), \\
& I_{1, \beta}(-\widetilde{B})=0.35165 \beta+(1-\beta)(0.4033) .
\end{aligned}
$$

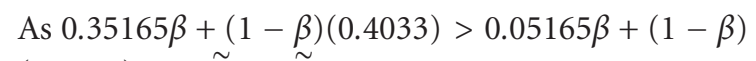$$
(0.4033) \Rightarrow-\widetilde{A}<-\widetilde{B}
$$

(iii) For a neutral decision maker,

$$
\begin{aligned}
& I_{0.5, \beta}(-\widetilde{A})=0.05165 \beta+(1-\beta)(0.05165), \\
& I_{0.5, \beta}(-\widetilde{B})=0.35165 \beta+(1-\beta)(0.35165) . \\
& \text { As } 0.35165 \beta+(1-\beta)(0.35165)>0.05165 \beta+(1-\beta) \\
& (0.05165) \Rightarrow-\widetilde{A}<-\widetilde{B} .
\end{aligned}
$$

Thus, we see that the ranking order is same in all the three cases.

From Examples 9 and 10, we see that $\widetilde{A}>\widetilde{B} \Rightarrow-\widetilde{A}<-\widetilde{B}$.

Example 11. Let $\widetilde{A}=(0.1,0.3,0.3,0.5 ; 0.8), \widetilde{B}=(0.1,0.3,0.3$, $0.5 ; 1)$.

Then, $S_{\widetilde{A}}\left(\overline{x_{0}}, \overline{y_{0}}\right)=(0.3,0.2533)$ and $S_{\widetilde{B}}\left(\overline{x_{0}}, \overline{y_{0}}\right)=(0.3$, $0.4033)$.

Therefore, $R(\widetilde{A})=0.3926$ and $R(\widetilde{B})=0.5026 \Rightarrow \widetilde{A}<\widetilde{B}$.

It is observed that the above ranking order is unaltered even by using the index of modality proposed in Definition 6 irrespective of decision maker. 
TABLE 1: Comparison of various ranking methods.

\begin{tabular}{|c|c|c|c|c|c|c|}
\hline Method & & $A_{1}$ & $A_{2}$ & $A_{3}$ & $A_{4}$ & Ranking order \\
\hline Yager & & 0.20 & 0.50 & 0.50 & 0.70 & $A_{4}>A_{2}=A_{3}>A_{1}$ \\
\hline Fortemps and Roubens & & 0.20 & 0.50 & 0.50 & 0.70 & $A_{4}>A_{2}=A_{3}>A_{1}$ \\
\hline \multirow{3}{*}{ Liou and Wang } & $\alpha=1$ & 0.25 & 0.65 & 0.65 & 0.75 & $A_{4}>A_{2}=A_{3}>A_{1}$ \\
\hline & $\alpha=0.5$ & 0.20 & 0.50 & 0.50 & 0.70 & $A_{4}>A_{2}=A_{3}>A_{1}$ \\
\hline & $\alpha=0$ & 0.15 & 0.35 & 0.35 & 0.65 & $A_{4}>A_{2}=A_{3}>A_{1}$ \\
\hline \multirow{3}{*}{ Chen and Lu [37] } & $\beta=1$ & -0.20 & 0.00 & 0.00 & -0.20 & $A_{2}=A_{3}>A_{1}=A_{4}$ \\
\hline & $\beta=0.5$ & -0.20 & 0.00 & 0.00 & -0.20 & $A_{2}=A_{3}>A_{1}=A_{4}$ \\
\hline & $\beta=0$ & -0.20 & 0.00 & 0.00 & -0.20 & $A_{2}=A_{3}>A_{1}=A_{4}$ \\
\hline
\end{tabular}

\section{Comparative Study}

Example 12. Consider two fuzzy numbers $A=(1,4,5)$ and $B=(2,3,6)$.

By Liou and Wang method [24], it is clear that the two fuzzy numbers are equal for all the decision maker's as $I_{T}^{\alpha}(A)=4.5 \alpha+(1-\alpha) 2.5$ and $I_{T}^{\alpha}(B)=4.5 \alpha+(1-\alpha) 2.5$ which is not even true by intuition. By using our method we have

$$
\begin{gathered}
S(A)=(3.6666,-0.5833), \quad S(B)=(3.3333,-0.5833), \\
R(A)=3.7127, \quad R(B)=3.3839 \Longrightarrow A>B .
\end{gathered}
$$

It is observed that the above ranking order is unaltered even by using the index of modality proposed in Definition 6 from the decision maker's view point.

Example 13. Consider four fuzzy numbers $A_{1}=(0.1,0.2$, $0.3 ; 1), A_{2}=(0.2,0.5,0.8 ; 1), A_{3}=(0.3,0.4,0.9 ; 1), A_{4}=$ $(0.6,0.7,0.8 ; 1)$ which were ranked earlier by Yager [10], Fortemps and Roubens [27], Liou and Wang [24], and Chen and $\mathrm{Lu}$ [37] as shown in Table 1.

It can be seen from Table 1 that none of the methods discriminates fuzzy numbers. Yager [10] and Fortemps and Roubens [27] methods failed to discriminate the fuzzy numbers $A_{2}$ and $A_{3}$, whereas the methods of Liou and Wang [24] and Chen and $\mathrm{Lu}$ [37] cannot discriminate the fuzzy numbers $A_{2}, A_{3}$ and $A_{1}, A_{4}$.

By using our method, we have

$$
\begin{array}{r}
S\left(A_{1}\right)=(0.2,0.4133), \quad S\left(A_{2}\right)=(0.5,0.3866), \\
S\left(A_{3}\right)=(0.4666,0.4), \quad S\left(A_{4}\right)=(0.7,0.4133) ; \\
R\left(A_{1}\right)=0.4591, \quad R\left(A_{2}\right)=0.6320, \\
R\left(A_{3}\right)=0.6146, \\
R\left(A_{4}\right)=0.8129 \Longrightarrow A_{4}>A_{2}>A_{3}>A_{1} .
\end{array}
$$

It is observed that the above ranking order is unaltered even by using the index of modality proposed in Definition 6 from the decision maker's view point.
Example 14. Let $\widetilde{A}=(0.1,0.2,0.4,0.5 ; 1)$ and $\widetilde{B}=(1,1,1$, $1 ; 1)$. Cheng [28] proposed a ranking function which is the distance from centroid point and the original point where as Chu and Tsao [39] proposed a ranking function which is the area between the centroid point and original point. Their centroid formulae are given by

$$
\begin{gathered}
\left(\frac{w\left(d^{2}-2 c^{2}+2 b^{2}-a^{2}+d c-a b\right)+3\left(c^{2}-b^{2}\right)}{3 w(d-c+b-a)+6(c-b)},\right. \\
\left.\frac{w}{3}\left(1+\frac{(b+c)-(a+d)(1-w)}{(b+c-a-d)+2(a+d) w}\right)\right), \\
\left(\frac{w\left(d^{2}-2 c^{2}+2 b^{2}-a^{2}+d c-a b\right)+3\left(c^{2}-b^{2}\right)}{3 w(d-c+b-a)+6(c-b)},\right. \\
\left.\frac{w}{3}\left(1+\frac{b+c}{a+b+c+d}\right)\right) .
\end{gathered}
$$

Both these Centroid formulae cannot rank crisp numbers which are a special case of fuzzy numbers as it can be seen from the above formulae that the denominator in the first coordinate of their Centroid formulae is zero, and hence Centroid of crisp numbers are undefined for their formulae. By using our method, we have

$$
\begin{gathered}
S_{\widetilde{A}}\left(\overline{x_{0}}, \overline{y_{0}}\right)=(0.3,0.3633), \\
S_{\widetilde{B}}\left(\overline{x_{0}}, \overline{y_{0}}\right)=(1,0.4166) .
\end{gathered}
$$

Therefore, $R(\widetilde{A})=0.4711$ and $R(\widetilde{B})=1.0833 \Rightarrow \widetilde{A}<\widetilde{B}$.

It is observed that the above ranking order is unaltered even by using the index of modality proposed in Definition 6 irrespective of decision maker choice.

\section{Conclusions}

This paper proposes a method that ranks fuzzy numbers which is simple and concrete. This method ranks trapezoidal as well as triangular fuzzy numbers and their images. This method also ranks crisp numbers which are special case of fuzzy numbers whereas methods proposed by Cheng and Chu cannot rank crisp numbers as theirpagebreak centroid 
formulae are undefined for crisp numbers. This method uses an index of modality which represents the importance of central value against the extreme values, beside the decision maker's degree of optimism. This method which is simple in calculation not only gives satisfactory results to well-defined problems, but also gives a correct ranking order to problems, whereas Yager index, Fortemps and Roubens, Liou and Wang, and Chen and Lu indexes failed to discriminate fuzzy numbers, and this method also agrees with human intuition.

\section{References}

[1] L. A. Zadeh, "Fuzzy sets," Information and Control, vol. 8, no. 3, pp. 338-353, 1965.

[2] R. Jain, "Decision making in the presence of fuzzy variables," IEEE Transactions on Systems, Man and Cybernetics, vol. 6, no. 10, pp. 698-703, 1976.

[3] G. Bortolan and R. Degani, "A review of some methods for ranking fuzzy subsets," Fuzzy Sets and Systems, vol. 15, no. 1, pp. 1-19, 1985.

[4] S. M. Baas and H. Kwakernaak, "Rating and ranking of multiple-aspect alternatives using fuzzy sets," Automatica, vol. 13, no. 1, pp. 47-58, 1977.

[5] R. Jain, "A procedure for multi aspect decision making using fuzzy sets," International Journal of Systems Science, vol. 8, no. 1, pp. 1-7, 1978.

[6] R. R. Yager, "Ranking fuzzy subsets over the unit interval," in Proceedings of the IEEE Conference on Decision and Control (CDC '78), pp. 1435-1437, Albuquerque, NM, USA, August 1978.

[7] J. F. Baldwin and N. C. F. Guild, "Comparison of fuzzy sets on the same decision space," Fuzzy Sets and Systems, vol. 2, no. 3, pp. 213-231, 1979.

[8] R. R. Yager, "On choosing between fuzzy subsets," Kybernetes, vol. 9, no. 2, pp. 151-154, 1980.

[9] J. M. Adamo, "Fuzzy decision trees," Fuzzy Sets and Systems, vol. 4, no. 3, pp. 207-219, 1980.

[10] R. R. Yager, "A procedure for ordering fuzzy subsets of the unit interval," Information Sciences, vol. 24, no. 2, pp. 143-161, 1981.

[11] W. Chang, "Ranking of fuzzy utilities with triangular membership functions," Proceedings of International Conference on Policy Analysis and Systems, pp. 263-272, 1981.

[12] E. Kerre, "The use of fuzzy set theory in electrocardiological diagnostics," in Approximate Reasoning in Decision-Analysis, M. M. Gupta and E. Sanchez, Eds., pp. 277-282, NorthHolland Publishing, Amsterdam, The Netherlands, 1982.

[13] D. Dubois and H. Prade, "Ranking fuzzy numbers in the setting of possibility theory," Information Sciences, vol. 30, no. 3, pp. 183-224, 1983.

[14] S. Murakami, H. Maeda, and S. Imamura, "Fuzzy decision analysis on the development of centralized regional energy control system," in Proceedings of the IFAC on Fuzzy Information, Knowledge Representation and Decision Analysis, pp. 353358, Marseille, France, 1983.

[15] S.-H. Chen, "Ranking fuzzy numbers with maximizing set and minimizing set," Fuzzy Sets and Systems, vol. 17, no. 2, pp. 113129, 1985.

[16] D. Dubois and H. Prade, "The mean value of a fuzzy number," Fuzzy Sets and Systems, vol. 24, no. 3, pp. 279-300, 1987.

[17] E. S. Lee and R.-J. Li, "Comparison of fuzzy numbers based on the probability measure of fuzzy events," Computers and
Mathematics with Applications, vol. 15, no. 10, pp. 887-896, 1988.

[18] M. Delgado, J. L. Verdegay, and M. A. Vila, "A procedure for ranking fuzzy numbers using fuzzy relations," Fuzzy Sets and Systems, vol. 26, no. 1, pp. 49-62, 1988.

[19] L. de Campos and G. A. Muñoz, "A subjective approach for ranking fuzzy numbers," Fuzzy Sets and Systems, vol. 29, no. 2, pp. 145-153, 1989.

[20] K. Kim and K. S. Park, "Ranking fuzzy numbers with index of optimism," Fuzzy Sets and Systems, vol. 35, no. 2, pp. 143-150, 1990.

[21] Y. Yuan, "Criteria for evaluating fuzzy ranking methods," Fuzzy Sets and Systems, vol. 43, no. 2, pp. 139-157, 1991.

[22] S. Heilpern, "The expected value of a fuzzy number," Fuzzy Sets and Systems, vol. 47, no. 1, pp. 81-86, 1992.

[23] J. J. Saade and H. Schwarzlander, "Ordering fuzzy sets over the real line: an approach based on decision making under uncertainty," Fuzzy Sets and Systems, vol. 50, no. 3, pp. 237246, 1992.

[24] T.-S. Liou and M.-J. Wang, "Ranking fuzzy numbers with integral value," Fuzzy Sets and Systems, vol. 50, no. 3, pp. 247255, 1992.

[25] F. Choobineh and H. Li, "An index for ordering fuzzy numbers," Fuzzy Sets and Systems, vol. 54, no. 3, pp. 287-294, 1993.

[26] P.-T. Chang and E. S. Lee, "Ranking of fuzzy sets based on the concept of existence," Computers and Mathematics with Applications, vol. 27, no. 9-10, pp. 1-21, 1994.

[27] P. Fortemps and M. Roubens, "Ranking and defuzzification methods based on area compensation," Fuzzy Sets and Systems, vol. 82, no. 3, pp. 319-330, 1996.

[28] C.-H. Cheng, "A new approach for ranking fuzzy numbers by distance method," Fuzzy Sets and Systems, vol. 95, no. 3, pp. 307-317, 1998.

[29] P. Anand Raj and D. Nagesh Kumar, "Ranking alternatives with fuzzy weights using maximizing set and minimizing set," Fuzzy Sets and Systems, vol. 105, no. 3, pp. 365-375, 1999.

[30] J.-S. Yao and K. Wu, "Ranking fuzzy numbers based on decomposition principle and signed distance," Fuzzy Sets and Systems, vol. 116, no. 2, pp. 275-288, 2000.

[31] X. Wang and E. E. Kerre, "Reasonable properties for the ordering of fuzzy quantities (I)," Fuzzy Sets and Systems, vol. 118, no. 3, pp. 375-385, 2001.

[32] X. Wang and E. E. Kerre, "Reasonable properties for the ordering of fuzzy quantities (II)," Fuzzy Sets and Systems, vol. 118, no. 3, pp. 387-405, 2001.

[33] X. Wang, A class of approaches to ordering alternatives, M.S. thesis, Taiyuan University Technology, 1987.

[34] W. Kołodziejczyk, "Orlovsky's concept of decision-making with fuzzy preference relation-further results," Fuzzy Sets and Systems, vol. 19, no. 1, pp. 11-20, 1986.

[35] K. Nakamura, "Preference relations on a set of fuzzy utilities as a basis for decision making," Fuzzy Sets and Systems, vol. 20, no. 2, pp. 147-162, 1986.

[36] M. Modarres and S.-S Nezhad, "Ranking fuzzy numbers by preference ratio," Fuzzy Sets and Systems, vol. 118, no. 3, pp. 429-436, 2001.

[37] L.-H. Chen and H.-W. Lu, "An approximate approach for ranking fuzzy numbers based on left and right dominance," Computers and Mathematics with Applications, vol. 41, no. 12, pp. 1589-1602, 2001.

[38] L. Tran and L. Duckstein, "Comparison of fuzzy numbers using a fuzzy distance measure," Fuzzy Sets and Systems, vol. 130, no. 3, pp. 331-341, 2002. 
[39] T.-C. Chu and C.-T. Tsao, "Ranking fuzzy numbers with an area between the centroid point and original point," Computers and Mathematics with Applications, vol. 43, no. 1-2, pp. 111-117, 2002.

[40] X.-W. Liu and S.-L. Han, "Ranking fuzzy numbers with preference weighting function expectations," Computers and Mathematics with Applications, vol. 49, no. 11-12, pp. 17311753, 2005.

[41] S. Abbasbandy and B. Asady, "Ranking of fuzzy numbers by sign distance," Information Sciences, vol. 176, no. 16, pp. 24052416, 2006.

[42] H. Sun and J. Wu, "A new approach for ranking fuzzy numbers based on fuzzy simulation analysis method," Applied Mathematics and Computation, vol. 174, no. 1, pp. 755-767, 2006.

[43] Y. Deng, Z. Zhenfu, and L. Qi, "Ranking fuzzy numbers with an area method using radius of gyration," Computers and Mathematics with Applications, vol. 51, no. 6-7, pp. 1127-1136, 2006.

[44] B. Asady and A. Zendehnam, "Ranking fuzzy numbers by distance minimization," Applied Mathematical Modelling, vol. 31, no. 11, pp. 2589-2598, 2007.

[45] S.-J. Chen and S.-M. Chen, "Fuzzy risk analysis based on the ranking of generalized trapezoidal fuzzy numbers," Applied Intelligence, vol. 26, no. 1, pp. 1-11, 2007.

[46] M. S. Garcia and M. T. Lamata, "A modification of the index of liou and wang for ranking fuzzy number," International Journal of Uncertainty, Fuzziness and Knowlege-Based Systems, vol. 15, no. 4, pp. 411-424, 2007.

[47] L.-W. Lee and S.-M. Chen, "Fuzzy risk analysis based on fuzzy numbers with different shapes and different deviations," Expert Systems with Applications, vol. 34, no. 4, pp. 2763-2771, 2008.

[48] Y.-J. Wang and H.-S. Lee, "The revised method of ranking fuzzy numbers with an area between the centroid and original points," Computers and Mathematics with Applications, vol. 55, no. 9, pp. 2033-2042, 2008

[49] C.-C. Chen and H.-C. Tang, "Ranking nonnormal ptrapezoidal fuzzy numbers with integral value," Computers and Mathematics with Applications, vol. 56, no. 9, pp. 2340-2346, 2008.

[50] E. Valvis, "A new linear ordering of fuzzy numbers on subsets of F (R)," Fuzzy Optimization and Decision Making, vol. 8, no. 2, pp. 141-163, 2009.

[51] S. Abbasbandy and T. Hajjari, "A new approach for ranking of trapezoidal fuzzy numbers," Computers and Mathematics with Applications, vol. 57, no. 3, pp. 413-419, 2009.

[52] Y.-M. Wang and Y. Luo, "Area ranking of fuzzy numbers based on positive and negative ideal points," Computers and Mathematics with Applications, vol. 58, no. 9, pp. 1769-1779, 2009.

[53] Z.-X. Wang, Y.-J. Liu, Z.-P. Fan, and B. Feng, "Ranking L$\mathrm{R}$ fuzzy number based on deviation degree," Information Sciences, vol. 179, no. 13, pp. 2070-2077, 2009.

[54] Y.-M. Wang, "Centroid defuzzification and the maximizing set and minimizing set ranking based on alpha level sets," Computers and Industrial Engineering, vol. 57, no. 1, pp. 228 236, 2009.

[55] S.-M. Chen and J.-H. Chen, "Fuzzy risk analysis based on ranking generalized fuzzy numbers with different heights and different spreads," Expert Systems with Applications, vol. 36, no. 3, pp. 6833-6842, 2009.

[56] D.-F. Li, "A ratio ranking method of triangular intuitionistic fuzzy numbers and its application to MADM problems,"
Computers and Mathematics with Applications, vol. 60, no. 6, pp. 1557-1570, 2010.

[57] Z.-X. Wang and Y.-N. Mo, "Ranking fuzzy numbers based on ideal solution," Fuzzy Information and Engineering, vol. 2, no. 1, pp. 27-36, 2010.

[58] B. Asady, "The revised method of ranking LR fuzzy number based on deviation degree," Expert Systems with Applications, vol. 37, no. 7, pp. 5056-5060, 2010. 

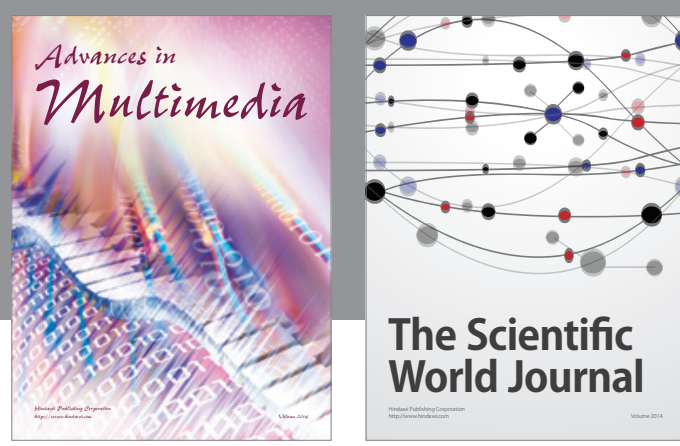

The Scientific World Journal
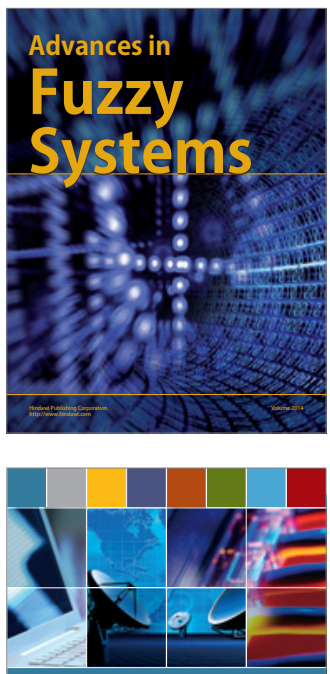

Computer Networks and Communications
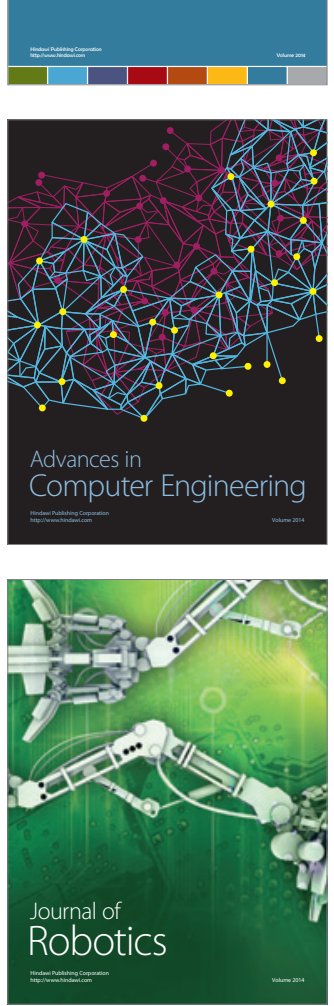
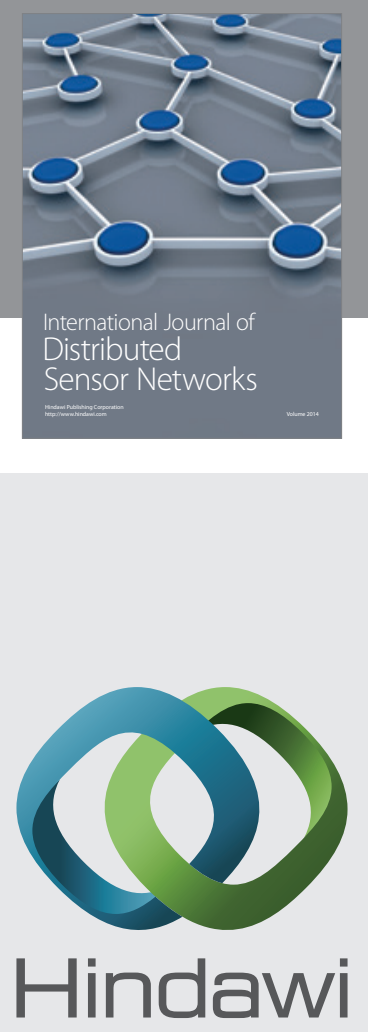

Submit your manuscripts at

http://www.hindawi.com
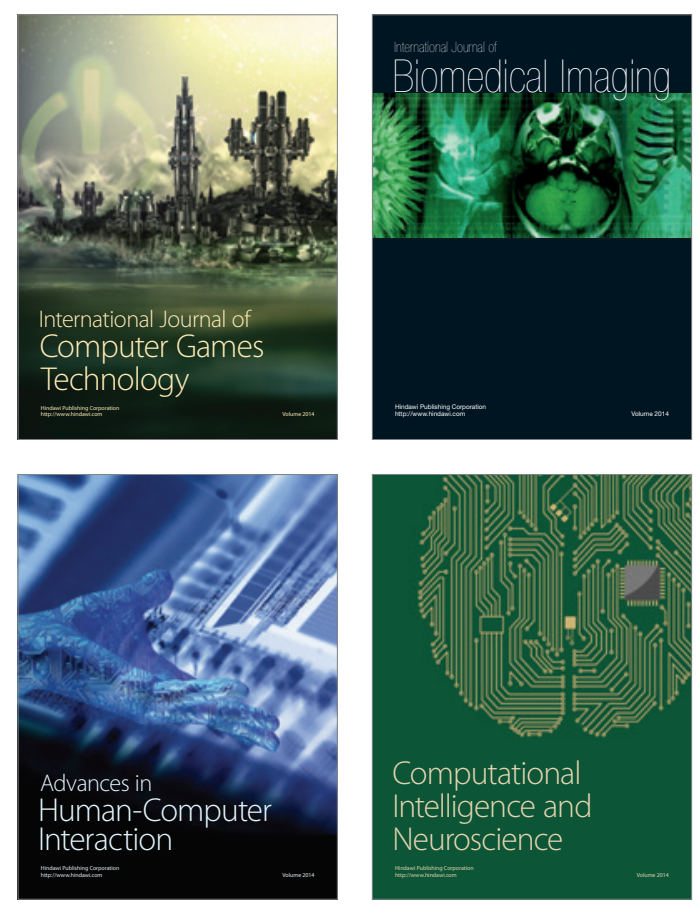
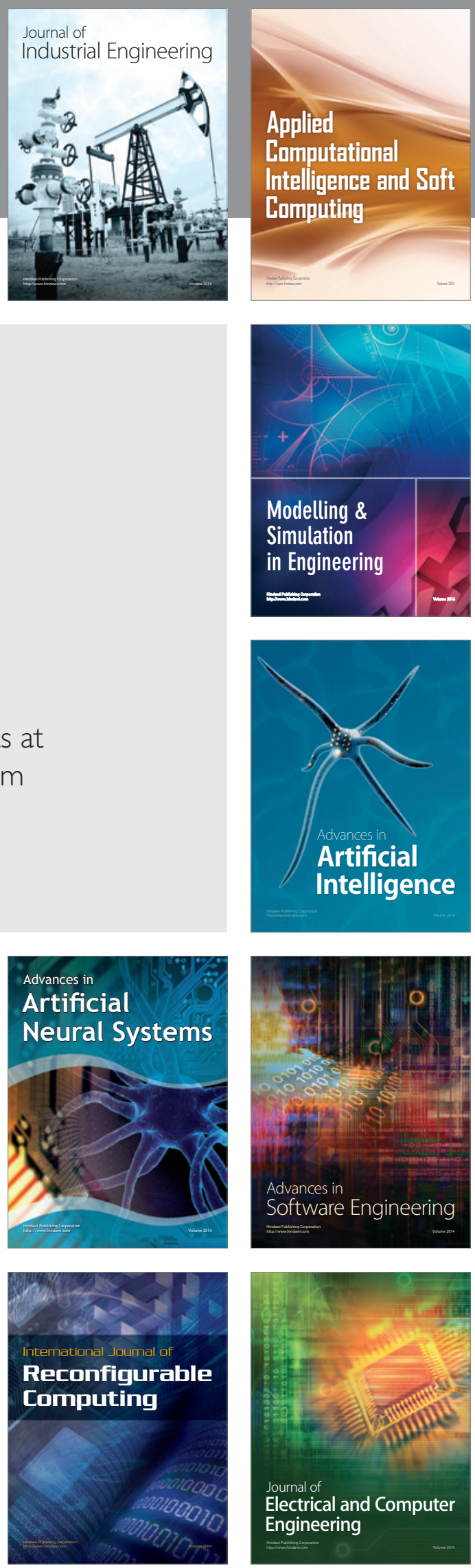